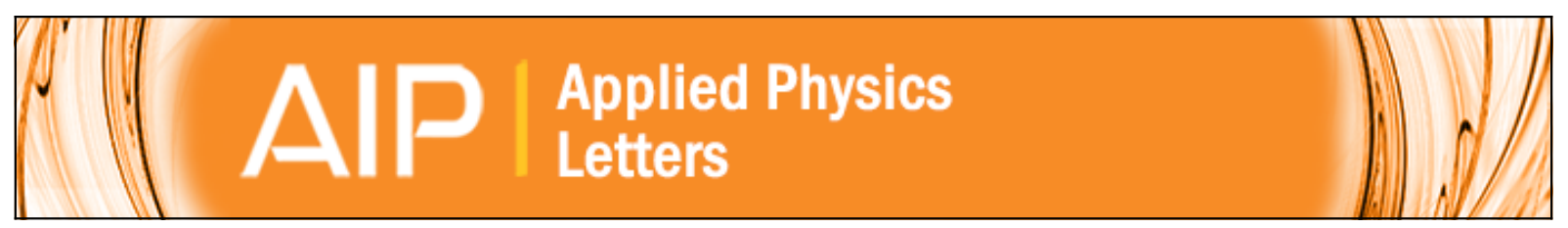

\title{
High gain single GaAs nanowire photodetector
}

Hao Wang

Citation: Applied Physics Letters 103, 093101 (2013); doi: 10.1063/1.4816246

View online: http://dx.doi.org/10.1063/1.4816246

View Table of Contents: http://scitation.aip.org/content/aip/journal/apl/103/9?ver=pdfcov

Published by the AIP Publishing

\section{Articles you may be interested in}

Large area, low capacitance, GaAs nanowire photodetector with a transparent Schottky collecting junction Appl. Phys. Lett. 103, 251109 (2013); 10.1063/1.4852136

GaAs nanowire Schottky barrier photovoltaics utilizing Au-Ga alloy catalytic tips

Appl. Phys. Lett. 101, 013105 (2012); 10.1063/1.4727907

Picosecond response times in GaAs/AlGaAs core/shell nanowire-based photodetectors

Appl. Phys. Lett. 98, 241113 (2011); 10.1063/1.3600061

Photocurrent and photoconductance properties of a GaAs nanowire

Appl. Phys. Lett. 95, 083111 (2009); 10.1063/1.3193540

Ultrafast and highly sensitive photodetectors fabricated on high-energy nitrogen-implanted GaAs

Appl. Phys. Lett. 83, 1719 (2003); 10.1063/1.1606879

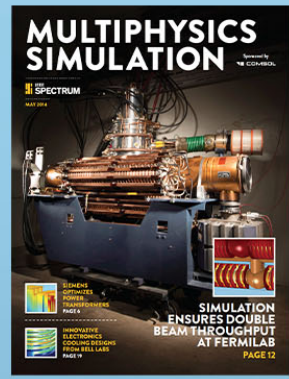

Free online magazine

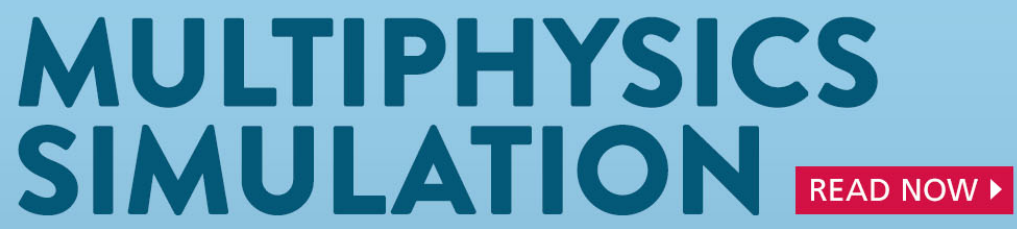




\title{
High gain single GaAs nanowire photodetector
}

\author{
Hao Wang ${ }^{\text {a) }}$ \\ Department of Electronic Materials Engineering, Research School of Physics and Engineering, \\ The Australian National University, Canberra, ACT 0200, Australia
}

(Received 10 February 2013; accepted 5 July 2013; published online 26 August 2013)

\begin{abstract}
An undoped single GaAs nanowire (NW) photodetector based on a metal-semiconductor-metal Schottky diode structure is fabricated by a focused ion beam method. The photoconductive gain of the device reaches 20000 at low laser excitation. Bias-dependence of gain proves that the surface contributes more to the gain at higher bias because of an increased surface charge region. The spectral response demonstrates not only the band-edge absorption profile of the single GaAs NW, but also the existence of leaky-mode resonance. (C) 2013 AIP Publishing LLC. [http://dx.doi.org/10.1063/1.4816246]
\end{abstract}

Metal-semiconductor-metal (M-S-M) structures have been employed to fabricate devices such as InGaAs photodetectors (PDs) for several decades. ${ }^{1,2}$ In such devices, a Schottky barrier forms at the metal/semiconductor interface and plays a crucial role in device performance. PDs based on the one-dimensional nanowire (NW) Schottky diode structure are of great interest because of the NWs size and associated physical properties. ${ }^{3-8}$ In NW PDs, Schottky barrier height is very sensitive to carrier generation and transport, ${ }^{7}$ and so controls the sensitivity of the PDs. ${ }^{8}$ Such PDs also show many advantages, for example, CdSe nanobelt PDs can be operated at high speed and photosensitivity, ${ }^{4} \mathrm{ZnS}$ PDs have the greatest potential as a UV detector within the UV-A band, ${ }^{5}$ and $\mathrm{ZnO}$ NW PDs have extremely high internal gain which results in a high gain-bandwidth product. ${ }^{6}$ Such superiorities are attributed to the high surface-to-volume ratio of the NWs (contributing to their high photosensitivity) and the reduced dimensions of the effective conductive channel (producing both high speed and high gain). ${ }^{6,8}$

Here, we report the fabrication and optoelectronic properties of an undoped single GaAs NW PD using the focused ion beam (FIB) technique and based on the back-to-back contact structure with $\mathrm{Pt}$ as the metal. Photoconductive gain up to 20000 with no observed saturation is achieved at $522 \mathrm{~nm}$ laser excitation. Both the band-edge absorption profile of the GaAs NW and their leaky-mode resonance are demonstrated and confirmed by simulation. The bias-dependences of gain and spectral response indicate that the surface plays a greater role in carrier transportation at higher bias.

Horizontal flow MOCVD was employed to grow undoped NWs at a pressure of 100 mbar and a total gas flow rate of $15 \mathrm{slm}$ using the two-temperature growth process. ${ }^{9}$ The NWs were grown on semi-insulating GaAs (111)B substrates using trimethylgallium (TMG) and arsine $\left(\mathrm{AsH}_{3}\right)$ precursors with $100 \mathrm{~nm}$ diameter Au nanoparticles as the catalyst. The obtained NWs had heights between 15 and $20 \mu \mathrm{m}$, diameters of $90-160 \mathrm{~nm}$, and a V/III ratio of 46 . After growth the NWs were separated from the substrate in an isopropanol solution by ultrasonication and then transferred on a photolithographically patterned Au-coated sapphire substrate. Pt was

\footnotetext{
${ }^{\text {a) }}$ Author to whom correspondence should be addressed. Electronic mail: wh-mouse@163.com
}

deposited on each end of a NW using a FIB system (FEI Helios 600 NANOLAB) at $28 \mathrm{pA} / 30 \mathrm{KV}$ to form Schottky contacts which were then connected to the $\mathrm{Au}$ electrodes. Current-voltage (IV) measurements were performed with a semiconductor parameter analyzer (HP4140). A pulsed laser operating at $1044 \mathrm{~nm}$ and frequency doubled to $522 \mathrm{~nm}$ with a pulse duration of $300 \mathrm{fs}$ and a repetition rate of $20 \mathrm{MHz}$ was used to photoexcite the device. After I-V measurements with laser injection, the laser beam was mechanically chopped at $280 \mathrm{~Hz}$ to induce a photocurrent with a digital lock-in amplifier. This setup was also used to characterize the polarization response by applying a $2.0 \mathrm{~V}$ bias across the NW. A xenon lamp $(1000 \mathrm{~W})$ with a digital lock-in amplifier was used as a continuous wavelength light source for light absorption measurements. The data from the lock-in amplifier and monochromator were collected with a computer and processed.

Fig. 1 shows an image of the device and its photocurrent mapping. The NW shown had a length of $16.1 \mu \mathrm{m}$ and diameter of $160 \mathrm{~nm}$. The $2 \mu \mathrm{m}$ tail of the FIB deposition beam could also be clearly observed (Fig. 1(a)), which means that the greatest part of the device was not contaminated during the process. The photocurrent mapping (Fig. 1(b)) exhibits large photocurrents at the contacts, which was due to diffusionlimited carrier transportation and local band-bending at the contact interfaces under bias (inset of Fig. 1(b)). ${ }^{10,11}$

Fig. 2(a) shows I-V curve of the device under laser excitation. The current increases with bias voltage with a bending characteristic in the voltage range of $-1 \mathrm{~V}$ to $1 \mathrm{~V}$, attributed to the back-to-back M-S-M Schottky diode structure. $^{8,12}$ It also reveals that photogenerated holes/electrons increased its conductivity under laser excitation due to photogating and photodopping effects. ${ }^{12}$ Fig. 2(a) also gives the corresponding photocurrent curves, which increase sharply in the $-1 \mathrm{~V}$ to $+1 \mathrm{~V}$ range and then reach saturation. This is because sufficient bias must be applied to obtain a flat band at the anode and separate the generated carriers. ${ }^{2}$

Fig. 2(b) displays photocurrent vs. absorbed excited laser power intensity. Considering the order of magnitude absorption difference between the absorption at $840 \mathrm{~nm}$ $(1.48 \mathrm{eV})$ and that at $522 \mathrm{~nm}(2.37 \mathrm{eV})$ from Fig. 3(a) and the $30 \%$ reflectance of the GaAs surface at $840 \mathrm{~nm},{ }^{13}$ the absorbance at $522 \mathrm{~nm}$ was estimated to be $7 \%$. Fig. 2(b) shows that the photocurrent increased exponentially with laser power, 
(a)

(b)
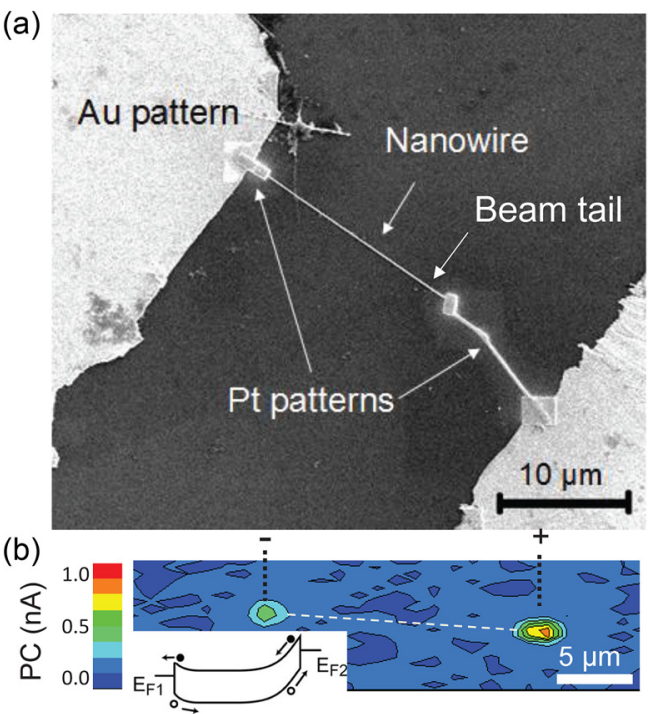

FIG. 1. (a) SEM image of a single GaAs NW back-to-back Schottky diode. Gold $(\mathrm{Au})$ patterns by photolithography and platinum (Pt) contacts by FIB process are indicated. (b) Photocurrent (PC) mapping image of the device with $2 \mathrm{~V}$ bias $\left(96 \mathrm{~W} \mathrm{~cm}^{-2}, 522 \mathrm{~nm}\right.$ laser). Inset: Energy band diagram and carrier transportation schematics of the device under bias.

while obeying the power law dependence $I \sim P^{a}$, where $a$ is $0.64,0.55$, and 0.52 for the biases of $2 \mathrm{~V}, 3 \mathrm{~V}$, and $4 \mathrm{~V}$, respectively. It is concluded that this decrease of $a$ was due to the effect of defects on carrier transportation, ${ }^{14}$ namely that higher bias caused more defects to become involved in carrier transportation. Further discussion on the contribution of surface defects is given below.

The photoconductive gain of the device under $522 \mathrm{~nm}$ laser excitation (Fig. 2(d)) reached 20000 at an absorption of $0.3 \mathrm{~W} / \mathrm{cm}^{2}$ and without saturation. The gain could be expected to be even higher at even lower laser excitation because the gain of $\mathrm{ZnO} \mathrm{NWs}{ }^{6}$ has been reported to reach $10^{8}$ when excited by a relatively low light power of $10 \mu \mathrm{W} /$ $\mathrm{cm}^{2}$. Because of the high surface-to-volume ratio of a NW, electrons ${ }^{6}$ or holes ${ }^{8}$ are trapped and this results in accumulation of the corresponding holes or electrons. ${ }^{15}$ In this experiment, light excited electrons moved to the surface of the undoped GaAs nanowire and were trapped, causing the accumulation of light excited holes. Thus, both of the electron trapping on the NW surface and the hole accumulation would affect the carrier drift and are the origin of the high gain observed. And since the absorption coefficient for extrinsic photoconductor is much lower than for intrinsic, ${ }^{16}$ it can be expected the gain would drop once the employed NW was intentionally doped. The gain observed in this work was also related to the excited laser intensity, and followed an inverse power law: $g \sim P^{-k} \cdot{ }^{17}$ Here, $k$ was $0.65,0.72$, and 0.89 for biases of $2 \mathrm{~V}, 3 \mathrm{~V}$, and $4 \mathrm{~V}$, respectively. This increase of $k$ with bias indicates that surface defects contributed more to carrier transportation; a $\mathrm{k}$ value close to 0.9 for the NW confirms that gain was dominated by the surface. ${ }^{6,8}$ Under higher bias the space charge region (SCR) becomes thicker, which decreases the effective cross-sectional area of carrier transportation paths through NW bulk section. In addition, the increased bias caused more electrons to be generated in NW bulk section and trapped on the surface, which
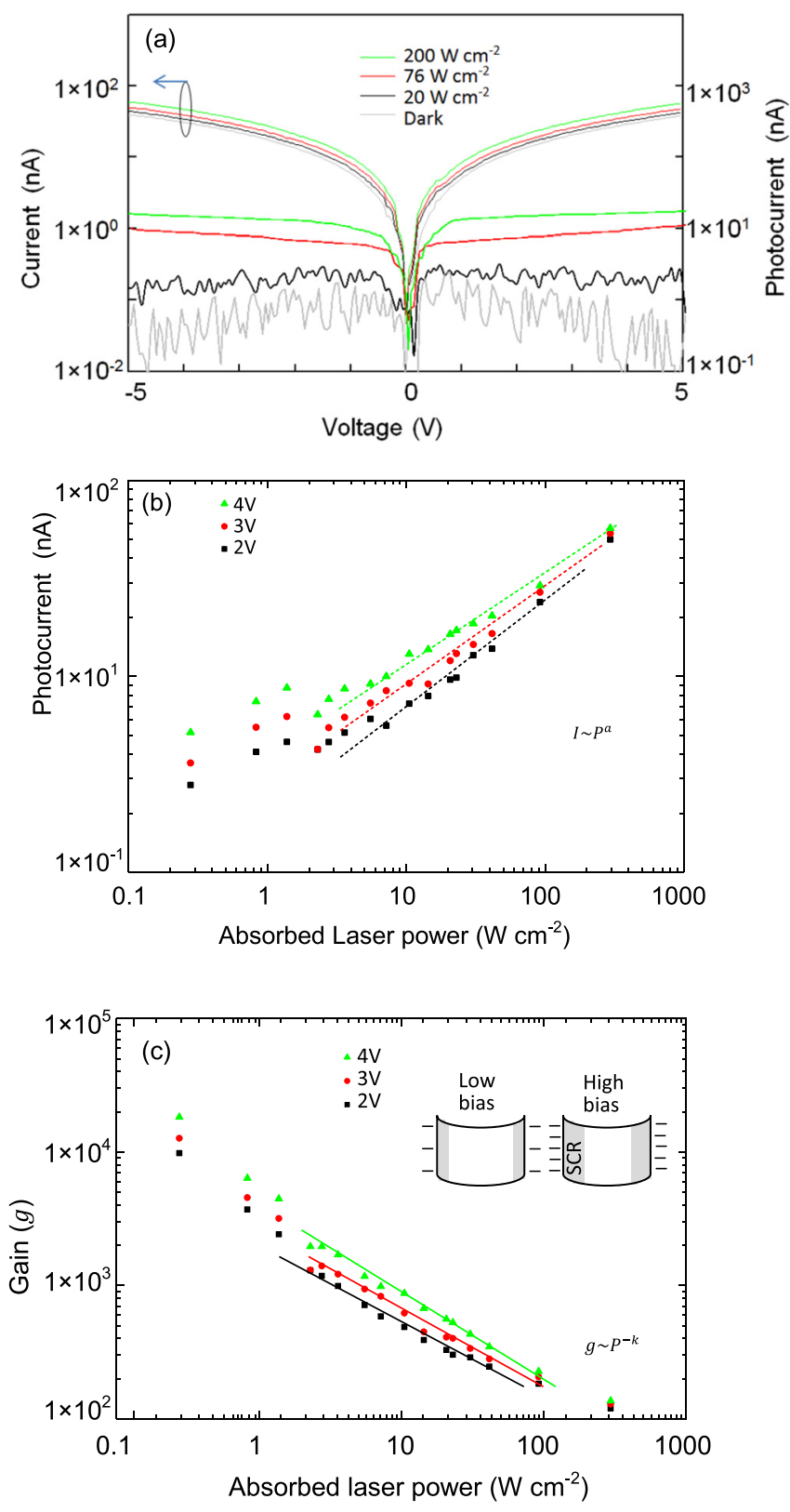

FIG. 2. (a) Current-voltage (I-V) curves and corresponding photocurrents of the device under $522 \mathrm{~nm}$ laser excitation at different power. (b) Power law dependence of photocurrent vs. absorbed laser power. Dashed lines indicate power law fittings, which exhibit exponents of $0.64,0.55$, and 0.52 for biases of $2 \mathrm{~V}, 3 \mathrm{~V}$, and $4 \mathrm{~V}$, respectively. (c) Photoconductive gain $(\mathrm{g})$ of the device under bias. Solid lines are fitted to $\boldsymbol{g} \sim \boldsymbol{P}^{-\boldsymbol{k}} . k$ is $0.65,0.72$, and 0.89 for biases of $2 \mathrm{~V}, 3 \mathrm{~V}$, and $4 \mathrm{~V}$, respectively. Insets show the SCRs of the NW under low and high biases.

increased the surface carrier density. Both of these effects, decrease of carrier transportation path and increase of surface carrier density, enhance the role of the surface in carrier transportation with bias increase. Thus, the greater contribution of the surface to the gain under higher bias is the reason for the observed $k$ increase. In addition, the gain at $0.07 \mathrm{~W} /$ $\mathrm{cm}^{2}$ increased from 10000 to 20000 when the bias was increased from $2 \mathrm{~V}$ to $4 \mathrm{~V}$. The increase of both $k$ and gain confirm that the surface has a greater effect on gain under higher bias. It can be expected the $k$ value would be lower if the NW is doped, since its SCR would be thinner compared with undoped NW. 


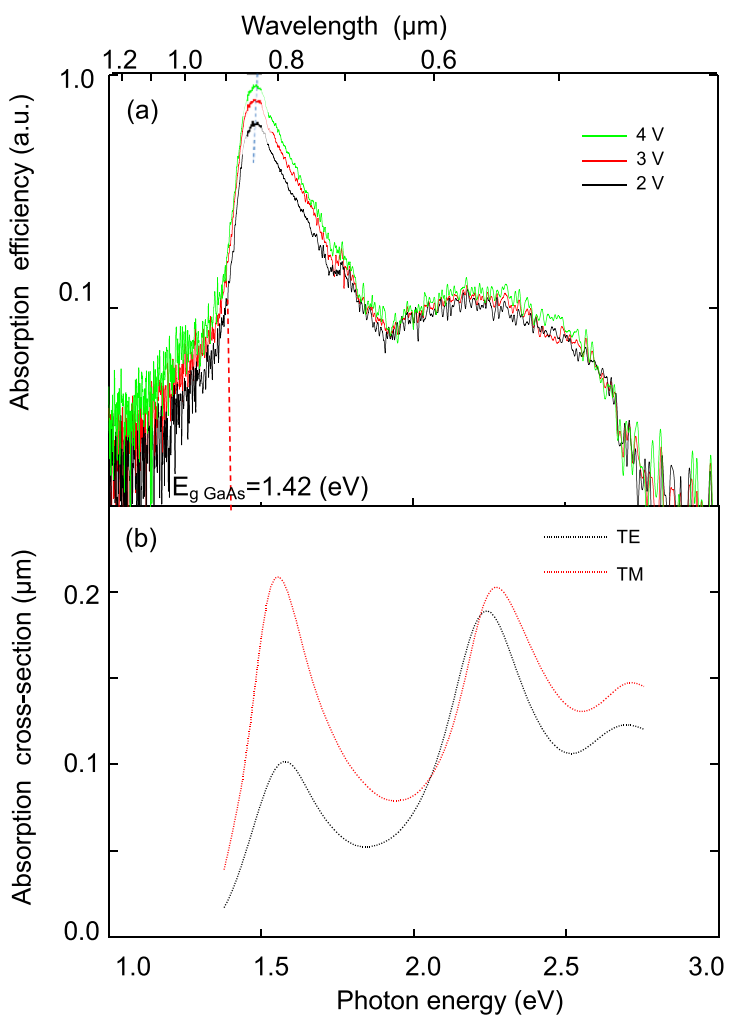

Power (a. u.)

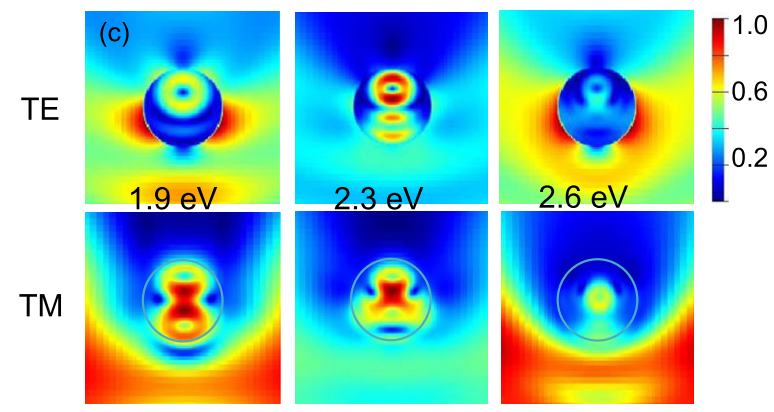

FIG. 3. (a) Spectral response of the device under different bias and xenon lamp (1000 W) excitation. Red dashed line is the bandgap of GaAs: $1.42 \mathrm{eV}$. Blue dashed line is the peak shift reference. (b) Simulated absorption efficiencies for a GaAs NW diameter of $160 \mathrm{~nm}$ taken using linearly polarized TE and TM light. (c) Simulated configuration of the power intensity for typical TE and TM modes for $1.9 \mathrm{eV}, 2.3 \mathrm{eV}$, and $2.6 \mathrm{eV}$, respectively. Each field is $400 \times 400 \mathrm{~nm}$ in size.
Fig. 3(a) plots the spectral response of the single NW device and shows two distinct peaks for the region investigated. The most sensitive peak at $1.5 \mathrm{eV}$ exhibited blue shift as well as increased absorption with bias. Such a blue shift could be attributed to the band-edge absorption of the GaAs NW. The signal increase was caused by the involvement of more carriers in the gain response due to the increase of carrier transportation rate with bias. In combination with the gain behavior observed under bias, it can therefore be concluded that under higher bias, the greater the amount of electron-hole pairs generated, the more the surface contributes to gain. The increased accumulation of carriers in the conduction and valence bands at higher bias caused the quasi-Fermi levels in the respective bands to move towards higher energy, resulting in the observed absorption peak blue shift.

The numerical simulations were performed for the peak in the $2.0 \mathrm{eV}$ to $2.5 \mathrm{eV}$ range using the finite-difference-timedomain (FDTD) technique with Lumerical FDTD Solutions. The resulting two-dimensional simulations modeled the GaAs NW as an ideal dielectric cylinder of infinite length, with complex refractive index (source Palik). A $160 \mathrm{~nm}$ diameter was used corresponding to the diameter of the NW in the fabricated device. The power absorbed $(\mathrm{W} / \mathrm{m})$ by the NW was determined under plane wave excitation for both transverse-electric (TE) (E vector perpendicular to NW axis) and transverse-magnetic (TM) (E vector parallel to NW axis) polarization, and in the range from $1.4 \mathrm{eV}$ to $2.75 \mathrm{eV}$. The total power absorbed divided by the source intensity $\left(\mathrm{W} / \mathrm{m}^{2}\right)$ gives the absorption cross-section (m). The field profiles across the cross-section of the NW were also obtained from the same simulations.

The results of the numerical simulations are shown in Fig. 3(b), where both TE and TM spectra exhibit another peak at higher energy level $(2.3 \mathrm{eV})$. In Fig. 3(c), the configuration of the power intensity for TE and TM modes of the respective energy levels clearly demonstrates that the electric field intensity for the resonance at $2.3 \mathrm{eV}$ was 2 times or even higher than that at $1.9 \mathrm{eV}$ and at $2.6 \mathrm{eV}$, for TE and TM modes, confirming that $550 \mathrm{~nm}(2.3 \mathrm{eV})$ is a leaky-mode resonant wavelength ${ }^{18}$ for GaAs NWs with a diameter of $160 \mathrm{~nm}$. The variation of the peak intensity at $2.3 \mathrm{eV}$ for the measured and simulated results is due to the discrepancy between the real NW geometry and the ideal design.
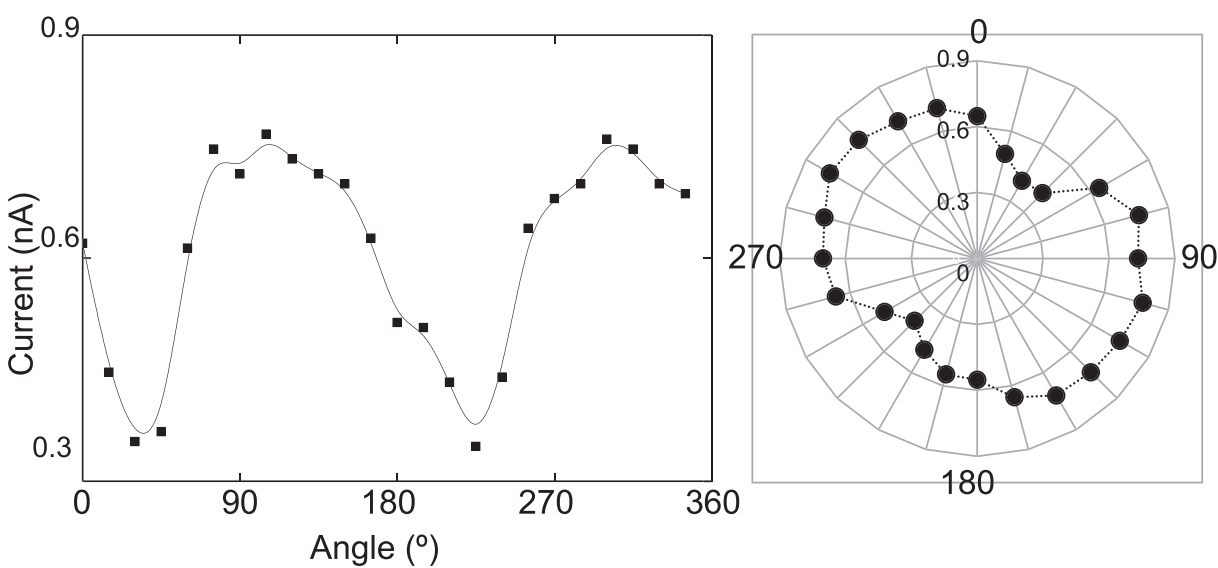

FIG. 4. Photocurrent polarization response under $2 \mathrm{~V}$ bias and $522 \mathrm{~nm}$ laser excitation with a power of $25 \mathrm{~mW} \mathrm{~cm}^{-2}$. 
Polarized photodetection of the single GaAs NW was also performed, and the results are given in Fig. 4. The dependence of the photocurrent signal on the polarization of the exciting light shows that the NW geometry strongly affected the polarization of emitted or absorbed photons and indicates that an individual NW is a suitable device element.

In summary, a M-S-M Schottky PD based on a single GaAs NW was fabricated using FIB and its optoelectronic properties were characterized. The surface exhibited photoconductive gain and greater spectral absorption under higher bias. At low laser excitation, a photoconductive gain of 20000 was achieved. The band-edge absorption profile and leaky-mode resonance of the GaAs NW was explored in detail. The present work paves a way to the fabrication of highly sensitive detectors based on single GaAs NWs.

The Australian Research Council is acknowledged for its financial support. Use of the fabrication facilities in this work was made possible through the Australian National Fabrication Facility. The author thanks Professor Chennupati Jagadish and Professor Hark Hoe Tan for discussions and their experimental support, and Mr. Dhruv Saxena for his simulation contribution.

${ }^{1}$ S. M. Sze, D. J. Coleman, and A. Loya, Solid-State Electron. 14, 1209 (1971).
${ }^{2}$ Julian B. D. Soole and Hermann Schumacher, IEEE J. Quantum Electron. 27, 737 (1991).

${ }^{3}$ J. Wang, M. S. Gudiksen, X. Duan, Y. Cui, and C. M. Lieber, Science 293, 1455 (2001)

${ }^{4}$ X. Jiang, Q. Xiong, S. Nam, F. Qian, Y. Li, and C. M. Lieber, Nano Lett. 7, 3214 (2007).

${ }^{5}$ Y. Liang, H. Liang, X. Xiao, and S. Hark, J. Mater. Chem. 22, 1199 (2012).

${ }^{6}$ C. Soci, A. Zhang, B. Xiang, S. A. Dayeh, D. P. Aplin, J. Park, X. Y. Bao, Y. H. Lo, and D. Wang, Nano Lett. 7, 1003 (2007).

${ }^{7}$ Z. Zhang, K. Yao, Y. Liu, C. Jin, X. Liang, Q. Chen, and L.-M. Peng, Adv. Funct. Mater. 17, 2478 (2007)

${ }^{8}$ C.-J. Kim, H.-S. Lee, Y.-J. Cho, K. Kang, and M.-H. Jo, Nano Lett. 10, 2043 (2010).

${ }^{9}$ H. J. Joyce, Q. Gao, H. H. Tan, C. Jagadish, Y. Kim, X. Zhang, Y. Guo, and J. Zhou, Nano Lett. 7, 921 (2007).

${ }^{10}$ J. K. Hyun and L. J. Lauhon, Nano Lett. 11, 2731 (2011).

${ }^{11}$ Y. Gu, J. P. Romankiewicz, J. K. David, J. L. Lensch, L. J. Lauhon, E. S. Kwak, and T. W. Odom, J. Vac. Sci. Technol. B 24, 2172 (2006).

${ }^{12}$ S. Thunich, L. Prechtel, D. Spirkoska, G. Abstreiter, A. Fontcuberta i Morral, and A. W. Holleitner, Appl. Phys. Lett. 95, 083111 (2009).

${ }^{13}$ H. R. Phillip and H. Ehrenreich, Phys. Rev. 129, 1550 (1963).

${ }^{14}$ S. C. Kung, W. Xing, W. E. can der Veer, F. Yang, K. C. Donavan, M. Cheng, J. C. Hemminger, and R. M. Penner, ACS Nano 5, 7627 (2011).

${ }^{15}$ T. Hanrath and B. A. Korgel, J. Phys. Chem. B 109, 5518 (2005).

${ }^{16} \mathrm{~A}$. Rogalski, Infrared Detectors, 2nd ed. (CRC Press, 2011).

${ }^{17}$ R.-S. Chen, H.-Y. Chen, C.-Y. Lu, K.-H. Chen, C.-P. Chen, L.-C. Chen, and Y.-J. Yang, Appl. Phys. Lett. 91, 223106 (2007).

${ }^{18}$ L. Cao, J. S. White, J.-S. Park, J. A. Schuller, B. M. Clemens, and M. L. Brongersma, Nature Mater. 8, 643 (2009). 